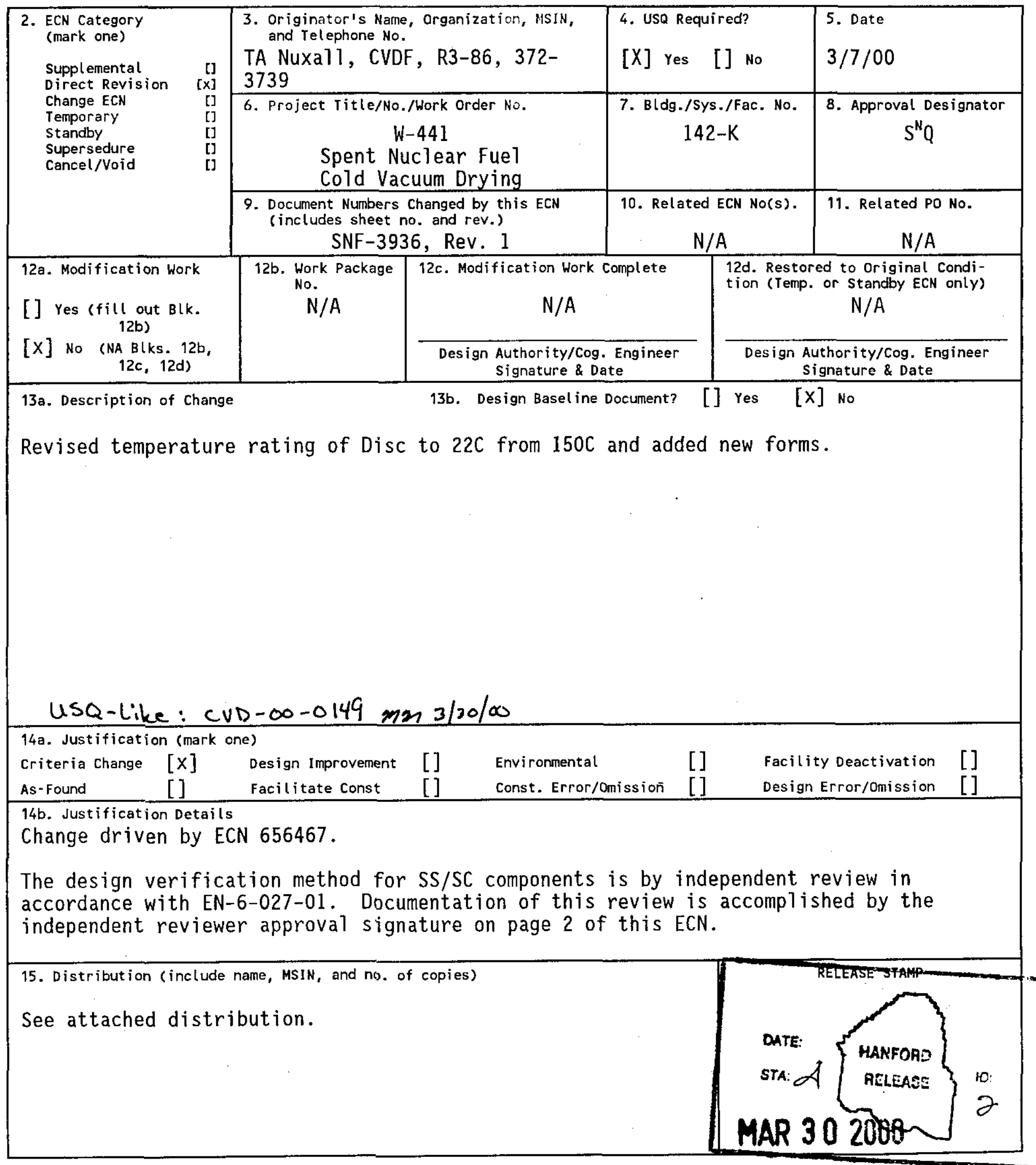




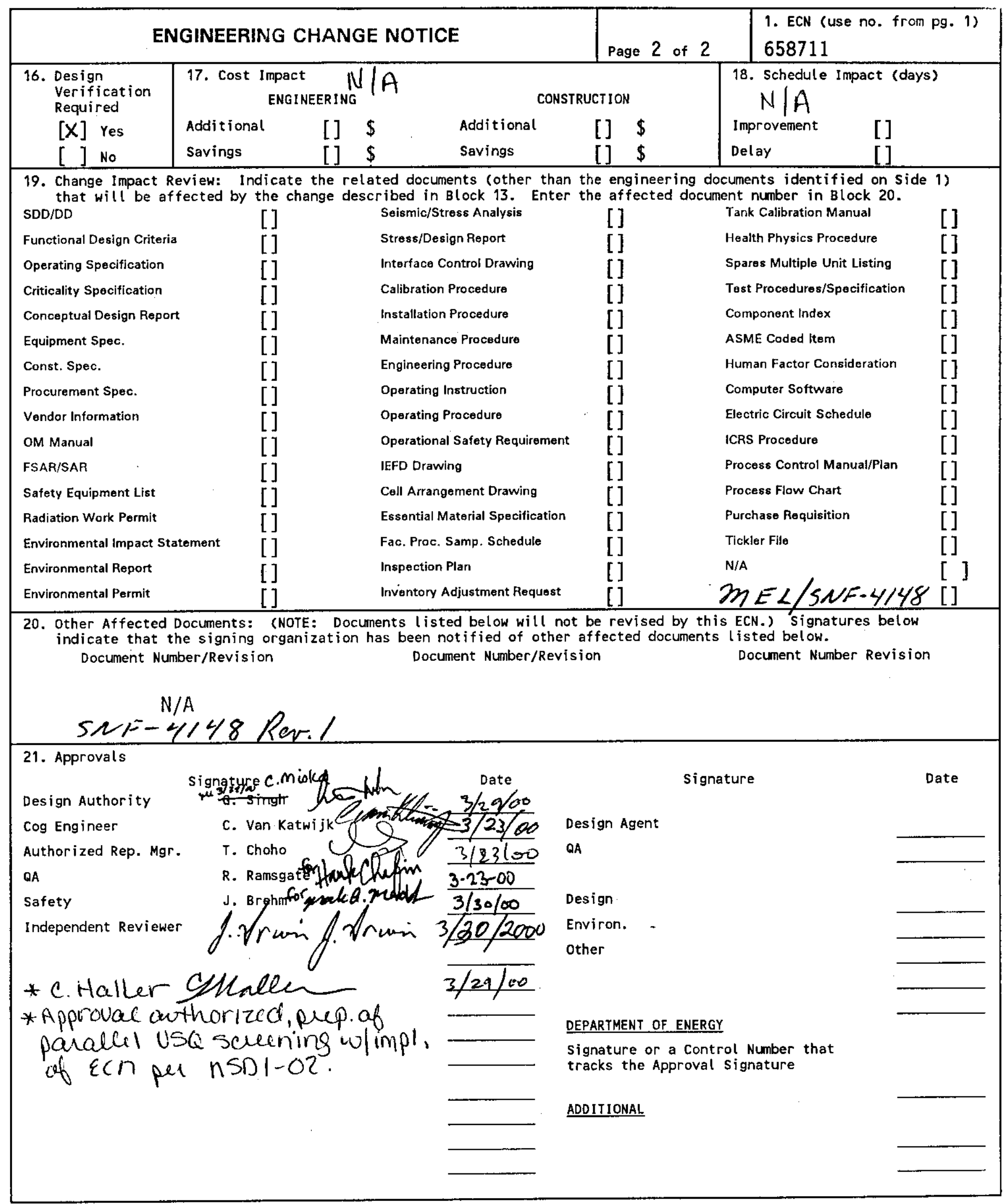


SNF-3936

Revision 2

\section{Fike MCO Pressure Safety Element}

Prepared for the U.S. Department of Energy

Assistant Secretary for Environmental Management

Project Hanford Management Contractor for the

U.S. Department of Energy under Contract DE-AC06-96RL.13200

Fluor Hanford

P.O. Box 1000

Richland, Washington 
TRADEMARK DISCLAIMER

Reference herein to any specific commercial product, process, or service by trade name, trademark, manufacturer, or

otherwise, does not necessarily constitute or imply its endorsement, recommendation, or favoring by the United

States Government or any agency thereof or its contractors or subcontractors.

This report has been reproduced from the best available copy. Available in paper copy and microfiche.

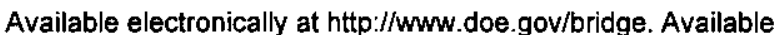
for a processing fee to the U.S. Department of Energy and its contractors, in paper, from:

U.S. Department of Energy

Office of Scientific and Technical Information

P.O. Box 62

Oak Ridge, TN 37831-0062

phone: 865-576-8401

fax: 865-576-5728

email: reports@adonis.osti.gov(423) 576-8401

Printed in the United States of America 
SNF-3936

Revision 2

10 dotal page.

\section{Fike MCO Pressure Safety Element}

Carl Van Katwijk

Fluor Hanford, Inc.

Date Published

March 3000

Prepared for the U.S. Department of Energy

Assistant Secretary for Environmental Management

Project Hanford Management Contractor for the

U.S. Department of Energy under Contract DE-AC06-96RL13200

\section{Fluor Hanford}

P.O. Box 1000

Richland, Washington
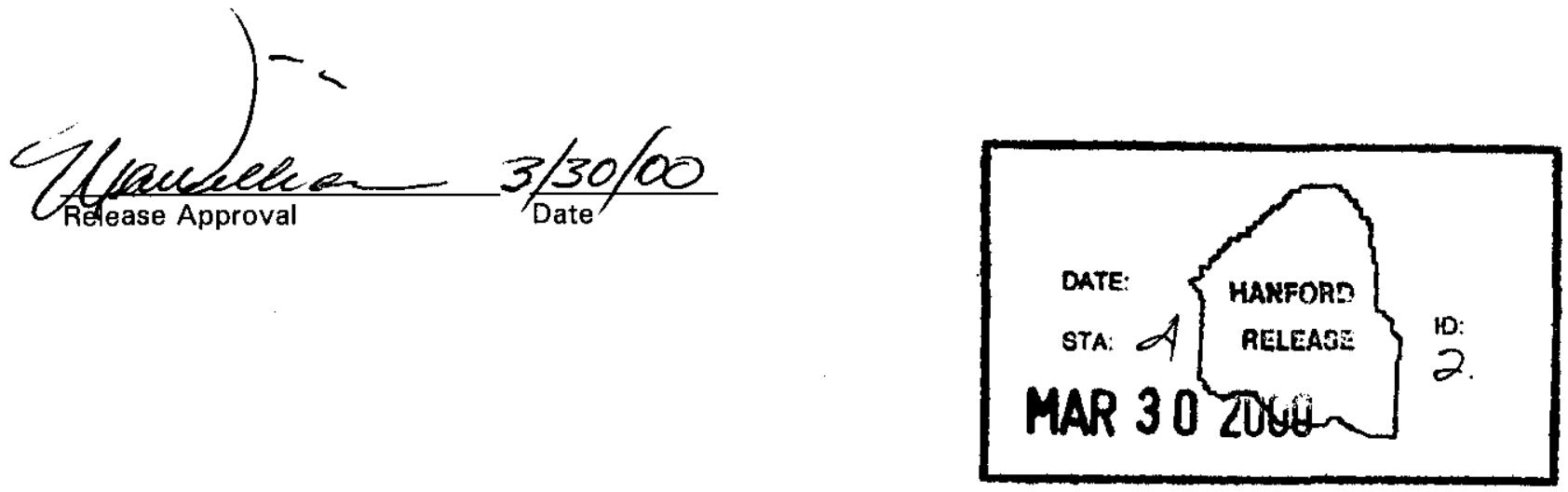


\section{RECORD OF REVISION}

\begin{tabular}{|l|l}
\hline $\begin{array}{l}\text { (1) Document Number } \\
\text { SNE-3936 }\end{array}$ & Page 1 \\
\hline
\end{tabular}

(2) Title

FIKE MCO PRESSURE SAEETY ELEMENT

Change Control Record

(3) Revision

0 EDT 626285, INITIALLY RELEASED

1

2 RS
(4) Description of Change - Replace, Add, and Delete Pages

ECN 653776, REVISION TO MEET SEL REV, 6

ECN 658711, REVISED TEMPERATURE RATING OF DISC TO 22C FROM 150C AND ADDED NEW FORMS.

Authorized for Release

\begin{tabular}{l|ll} 
(5) Cog. Engr. & (6) Cog. Mgr. Date
\end{tabular}

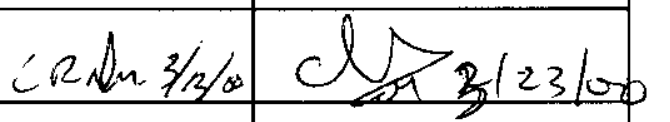




\section{Section 1 Pat Intermation}

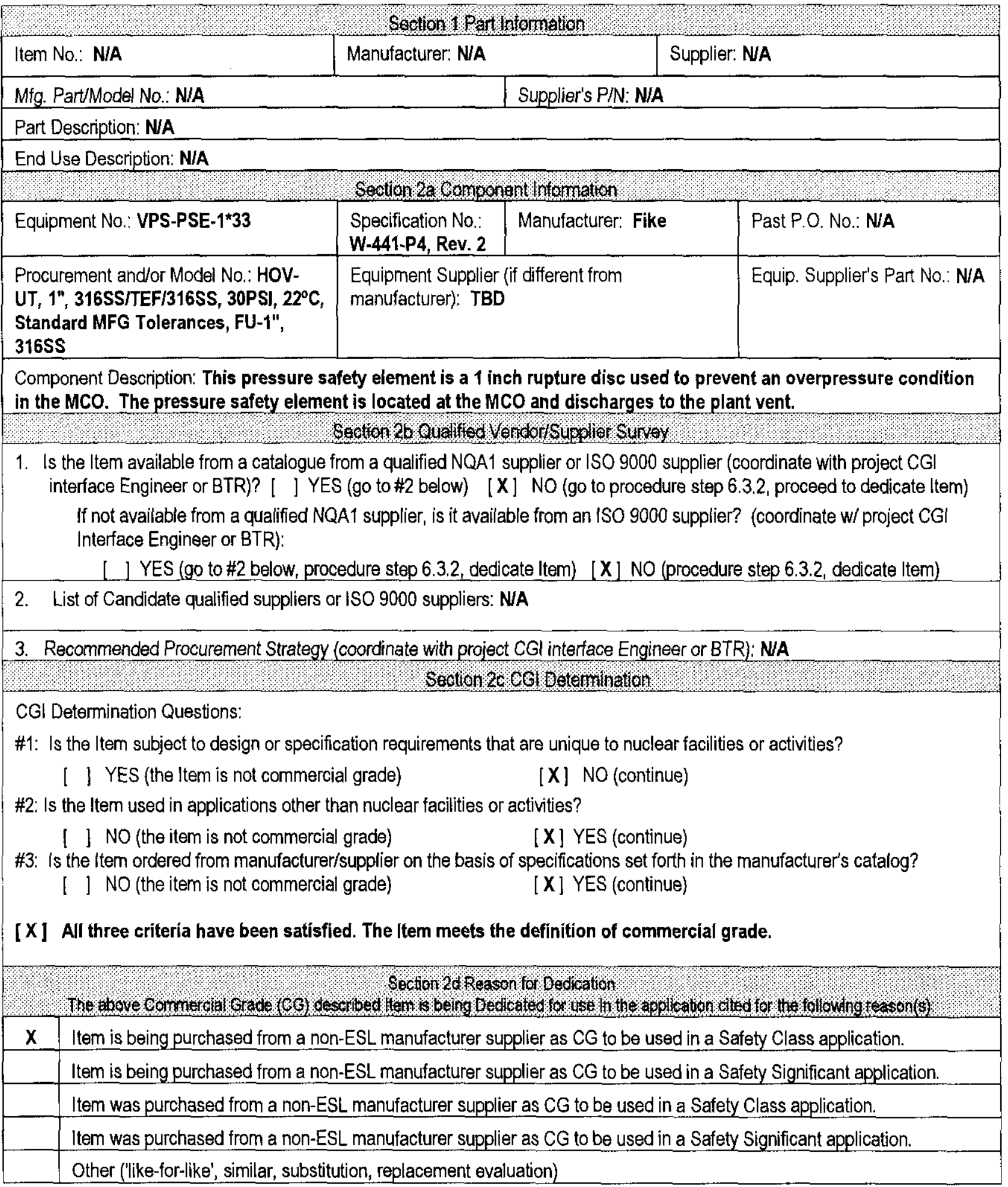




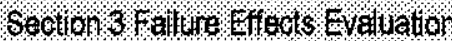

A. Part/Component Safety Function:

1. Maintains pressure boundary, prevents helium leakage from $M C O$ or air leakage into MCO during vacuum operation, $\mathrm{H} 2$ explosion.

2. Prevents MCO over pressure, prevents thermal runaway

3. Maintain critical function before and after seismic event.

B. Part/Component Functional Mode:

Safety Function \#1: [ ] Active [X] Passive

Safety Function \#2: [X] Active [ ] Passive

Safety Function \#3: [ ] Active [X] Passive

Active - Mechanical or Electrical change of state is required to occur for the component to perform its safety function

Passive - Change of state is not required for the component to perform its safety function

C. Host Component Safety Function (if applicable): N/A

1.

D. Failure Mechanisms(s) and the effects on component or system safety function (see worksheet 1):

1. Failure of the disc to rupture at its nominal burst pressure.

2. Fracture of the disc housing or piping attachment.

3. Disc ruptures or leaks at a lower pressure than its nominal burst pressure.

4. Upon actuation of rupture disc, pieces of disc separate and block downstream flow path and/or check valve. Section 4 Envirommental \& Natural Phenenent thazard Design

Environmental Qualification Required:

If yes: Environmental Qualification Requirements

Yes [ ]

Limiting Environmental Conditions:

No $[X]$

Required Safety Functions:

Environmental Condition $\mathrm{B}$ Qualification Period:

Natural Phenomena Hazard (NPH) Design Required:

Yes [X]

No [ ]

HNF-PRO-97, Rev. 0

If yes: NPH Design Requirements

Performance Category: PC-3

NPH Design Req'ts.: Seismic Condition A

Required Safety Functions: Disc ruptures to relieve

W-441-P4, Rev. 2 overpressure in the MCO. Pressure Boundary Integrity.

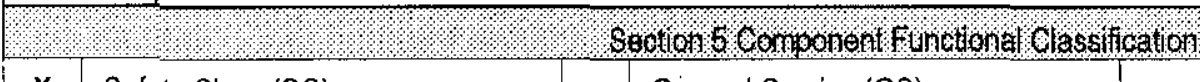

X Safety Class (SC)

General Service (GS)

Safety Significant (SS)

If part/component classification is different from host component/system, document basis. NA

\section{Sections 6 aind 7 [resened]}

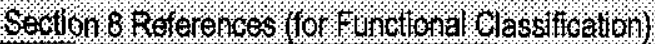

National Codes/Standards: ASME B31.3

Safety Analysis Report (SAR): HNF- 3553, Rev. 0, Annex B

Drawings: H-1-84010, Rev. 2, HNF-SD-SNF-SEL-002, Rev. 7

Vendor Manual/Manufacturer/Supplier Information: Fike Rupture Disc Application, Selection, and Specification, HOV Series 
Title: Fike Mco Pressure Safety Element

\begin{tabular}{|c|c|c|c|c|c|c|}
\hline 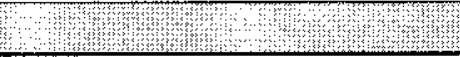 & \multicolumn{2}{|c|}{ section 9 orital Characteristics } & & & & \\
\hline Critical Characteristics & \multicolumn{2}{|c|}{ Acceptance Criteria/Tolerances } & \multicolumn{2}{|c|}{ Acc. Method } & ID & Function \\
\hline \multicolumn{7}{|c|}{ 1. Item Identification Critical Characteristics (necessary for reasonable assurance that the Item delivered is the Item specified) } \\
\hline $\begin{array}{l}\text { Component Number-Procedure } \\
\text { and/or Model Number }\end{array}$ & \multicolumn{2}{|c|}{$\begin{array}{l}\text { HOV-UT, 1", 316SSITEF/316SS, } 30 \text { PSI, } \\
22^{\circ} \mathrm{C} \text {, Standard MFG. Tolerance, FU-1", } \\
316 S \text {, (Per Procurement Package W-441- } \\
\text { P4, Rev. 2, Section H, Design Data Sheet) }\end{array}$} & \multicolumn{2}{|c|}{$1, \mathbf{I N}$} & $\mathrm{x}$ & \\
\hline Manufacturer & \multicolumn{2}{|c|}{ Fike } & \multicolumn{2}{|c|}{$1, \mathbb{I N}$} & $\underline{x}$ & \\
\hline Process Connection & \multicolumn{2}{|l|}{1 Inch Butt Weld } & \multicolumn{2}{|c|}{$1, \mathbf{I N}$} & $\mathrm{x}$ & \\
\hline \multicolumn{7}{|c|}{ 2. Physical Critical Characteristics (necessary for reasonable assurance that the ltem delivered is the Item specified) } \\
\hline Material, Housing & \multicolumn{2}{|c|}{ Stainless Steel (Note 4) } & \multicolumn{2}{|c|}{$1, \mathrm{IN}, 1, \mathrm{~T}$} & $\mathrm{X}$ & \\
\hline Material, Process Connection & \multicolumn{2}{|c|}{ Stainless Steel (Note 4) } & \multicolumn{2}{|c|}{$1, \mathrm{IN}, 1, \mathrm{~T}$} & $\mathrm{x}$ & \\
\hline Material, Rupture Disc & \multicolumn{2}{|c|}{ Teflon (Note 4) } & \multicolumn{2}{|c|}{$1, \mathbb{I N}, 1, T$} & $\mathrm{X}$ & \\
\hline \multicolumn{7}{|c|}{ 3. Performance Critical Characteristics (for reasonable assurance that the Item will perform its intended safety function(s)) } \\
\hline Pressure Boundary Integrity & \multicolumn{2}{|c|}{$\begin{array}{l}\text { No leakage at test pressure of } 165 \mathrm{psig} \\
\text { for }>10 \text { minutes. (Note } 3 \text { ) }\end{array}$} & \multicolumn{2}{|c|}{$1, T$} & & $\mathbf{x}$ \\
\hline Internal Pressure & \multicolumn{2}{|c|}{150 psig and Full Vacuum } & \multicolumn{2}{|l|}{$1, T$} & & $\mathbf{X}$ \\
\hline Leakage Past Rupture Disc & \multicolumn{2}{|c|}{$\begin{array}{l}\text { Pressure Test to } 15 \mathrm{psig} \text {, leakage to } \\
\text { bubble tight standards }<10-3 \mathrm{ml} / \mathrm{sec} \\
\text { (Note 5) }\end{array}$} & \multicolumn{2}{|c|}{$1, T$} & & $\mathbf{x}$ \\
\hline Rupture Disc Burst Pressure & \multicolumn{2}{|c|}{ Nominal 32 psig \pm 2 psig } & \multicolumn{2}{|c|}{$1, T$} & & $\underline{x}$ \\
\hline Inspect Disc after Rupture & \multicolumn{2}{|c|}{ No loose pieces from ruptured disc. } & \multicolumn{2}{|c|}{$1, T$} & & $x$ \\
\hline Environmental & \multicolumn{2}{|c|}{ Note 1} & & & & \\
\hline Seismic Condition A & Note 2 & & \multicolumn{2}{|c|}{$1, T$} & & $\mathbf{x}$ \\
\hline \multicolumn{4}{|c|}{$\begin{array}{l}\text { 4. Notes and Legend: } \\
\text { 1. The pressure safety element and rupture disc are not subject to degradation at environmental } \\
\text { conditions of } 40 \mathrm{~F} \text { and } 60 \% \text { RH or } 115^{\circ} \mathrm{F} \text { and } 22 \% \mathrm{RH} \text { and are suitable for Condition B application. } \\
\text { 2. Maintain critical function before and after Seismic event. W-441-P4-Rev. } 2 \text {, Appendix } \mathrm{L} \text {, page L-20 } \\
\text { provides a seismic testing plan for this safety class, Seismic Condition A component at a Seismic } \\
\text { Specta TBD. Equipment that has been shaker-table tested should not be installed in a plant (Ref. } \\
\text { IEEE Standard } 344-1984, \text { Section } 7 \text { ). Consequently, the seismic test constitutes a destructive test. } \\
\text { The industry sampling practice for destructive test is to test only one item. }\end{array}$} & \multicolumn{3}{|c|}{$\begin{array}{l}\text { Acceptance Method: } \\
\text { 1. Special Test and Inspection } \\
1, \mathrm{~N} \text { for Inspection } \\
\text { 1,T for Test } \\
\text { 2. Commercial Grade Survey } \\
\text { 3. Source Verification } \\
\text { 4. Vendoriltem History }\end{array}$} \\
\hline 4 & \multicolumn{3}{|c|}{ Section 10 nitial Reviews and Approvals } & & & \\
\hline \multicolumn{2}{|c|}{ 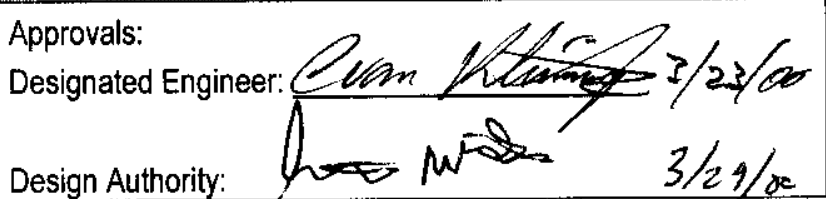 } & $\begin{array}{l}\text { QA Engineer: Ha } \\
\text { Other: }\end{array}$ & & & & \\
\hline
\end{tabular}




\section{WORKSHEET 1}

\section{DETERMINATION OF FAILURE MECHANISMS}

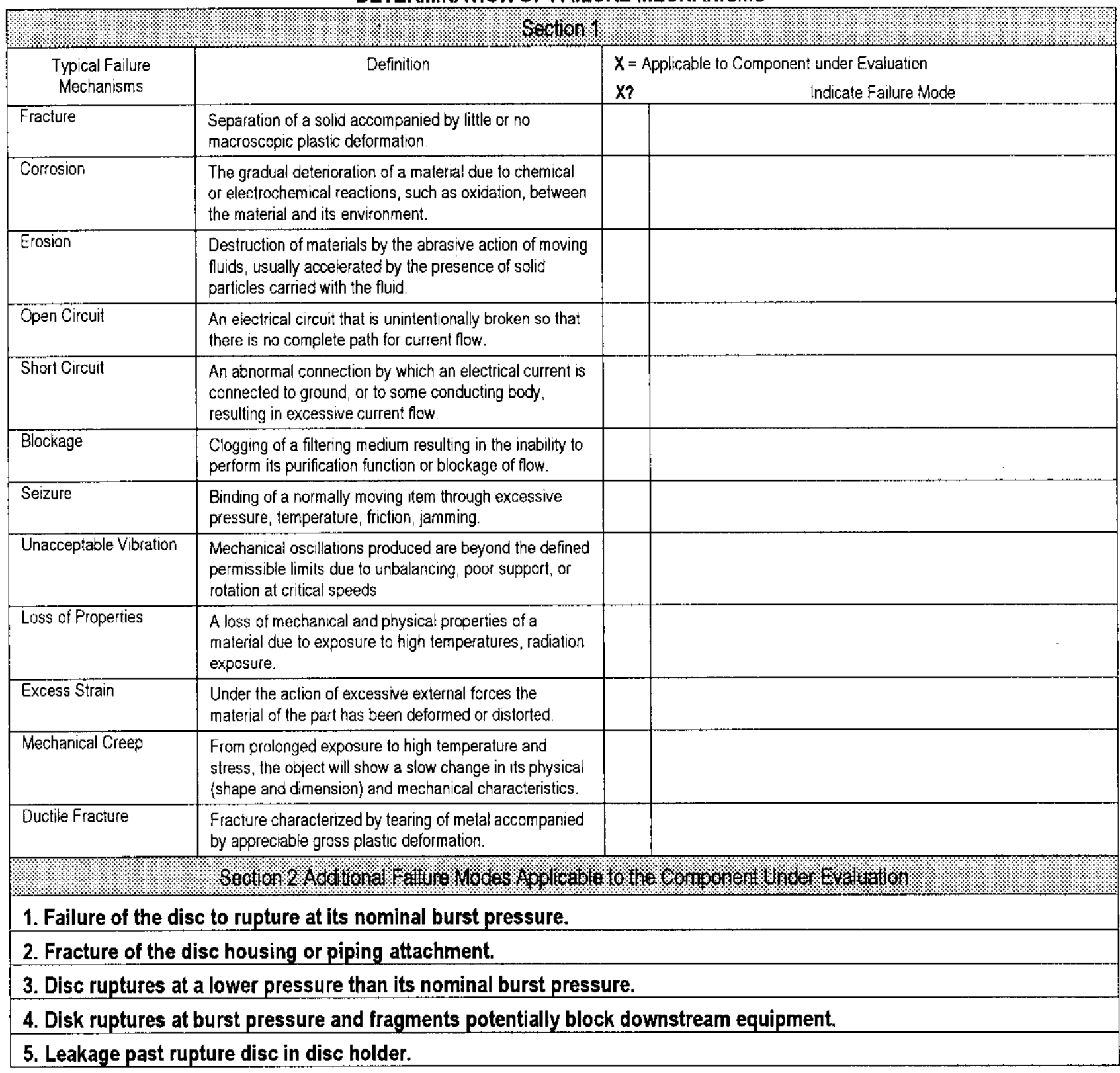


ECN No.NA CGI No. CGI-SNF-D-07-P4-044

SNF-3936, Rev. 2

Title: Fike Mco Pressure Safety Element

Checklist 1 - Acceptance Method 1 - Special Test/lnspection Verification

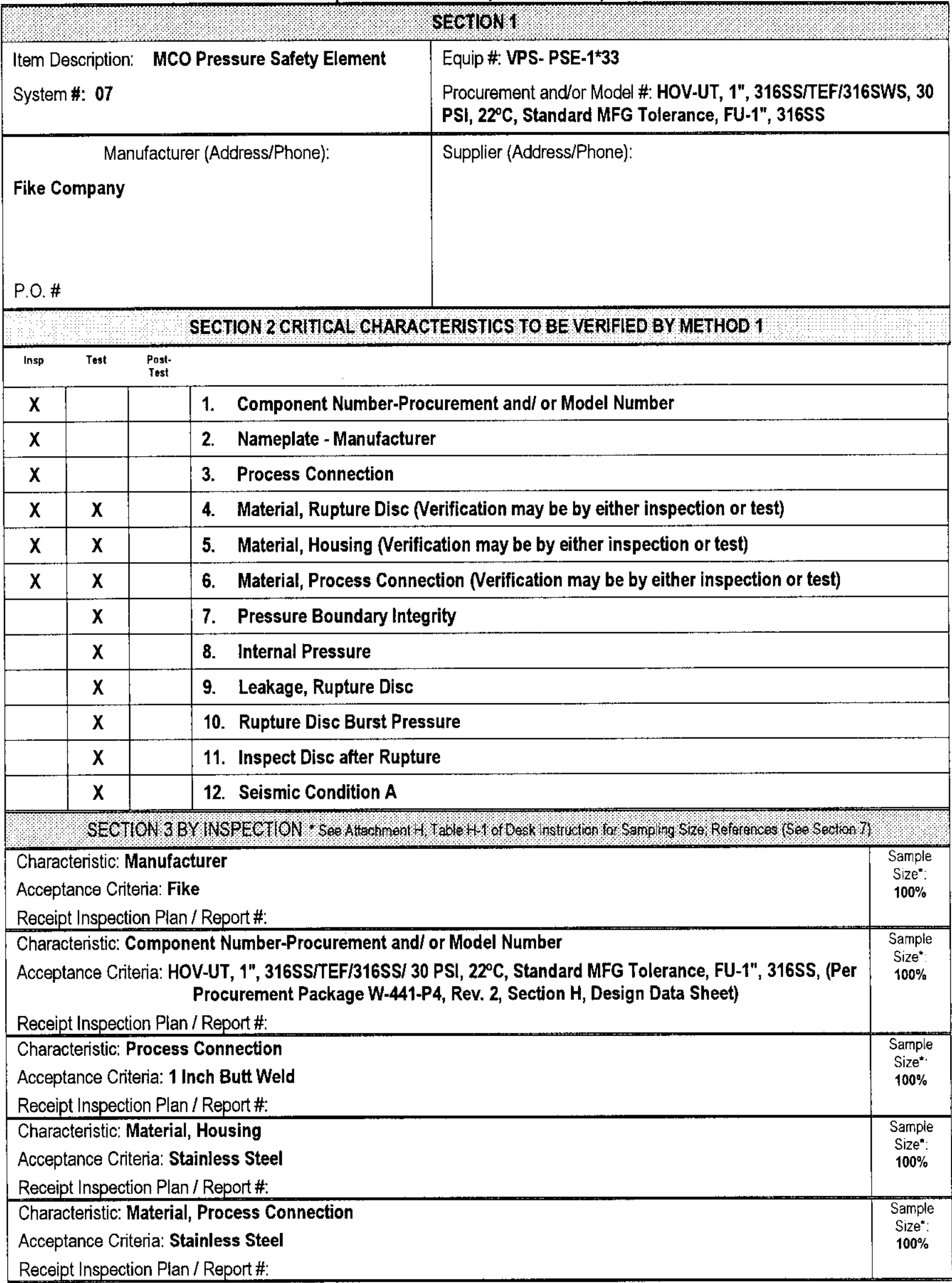




\begin{tabular}{|l|l|}
\cline { 2 - 2 } Commercial Grade Item Upgrade Dedication Form & SNF-3936, Rev. 2 \\
\hline $\begin{array}{l}\text { ECN No. NA CGI No._CGI-SNF.D-07-P4-044 } \\
\text { Titte: Fike Mco Pressure Safety Element }\end{array}$ & Page 6 of 8 \\
\hline
\end{tabular}

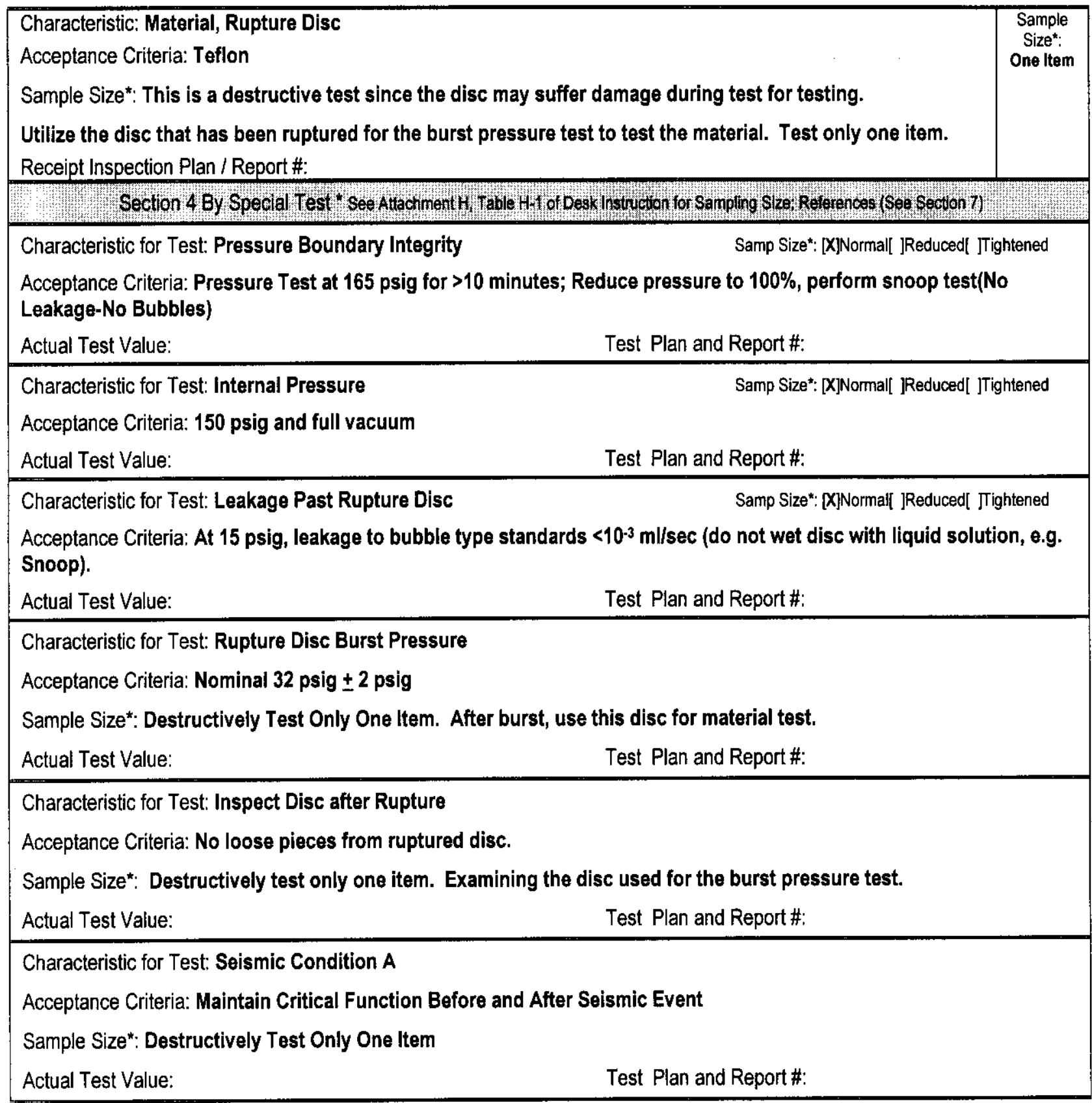

m*If Supplier/Manufacturer or Other, Refer to CG1 Checklist-2 for Support Information 


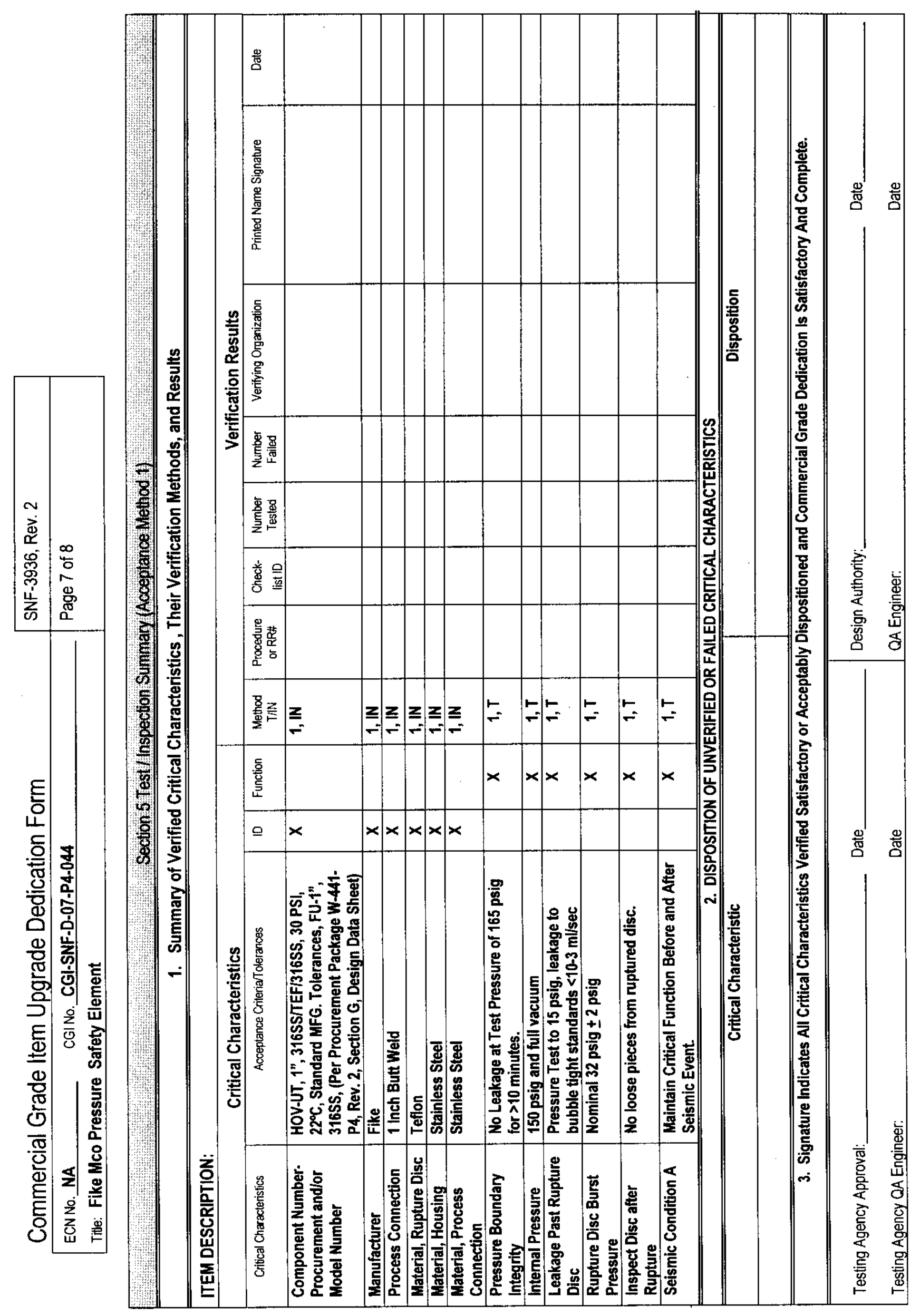




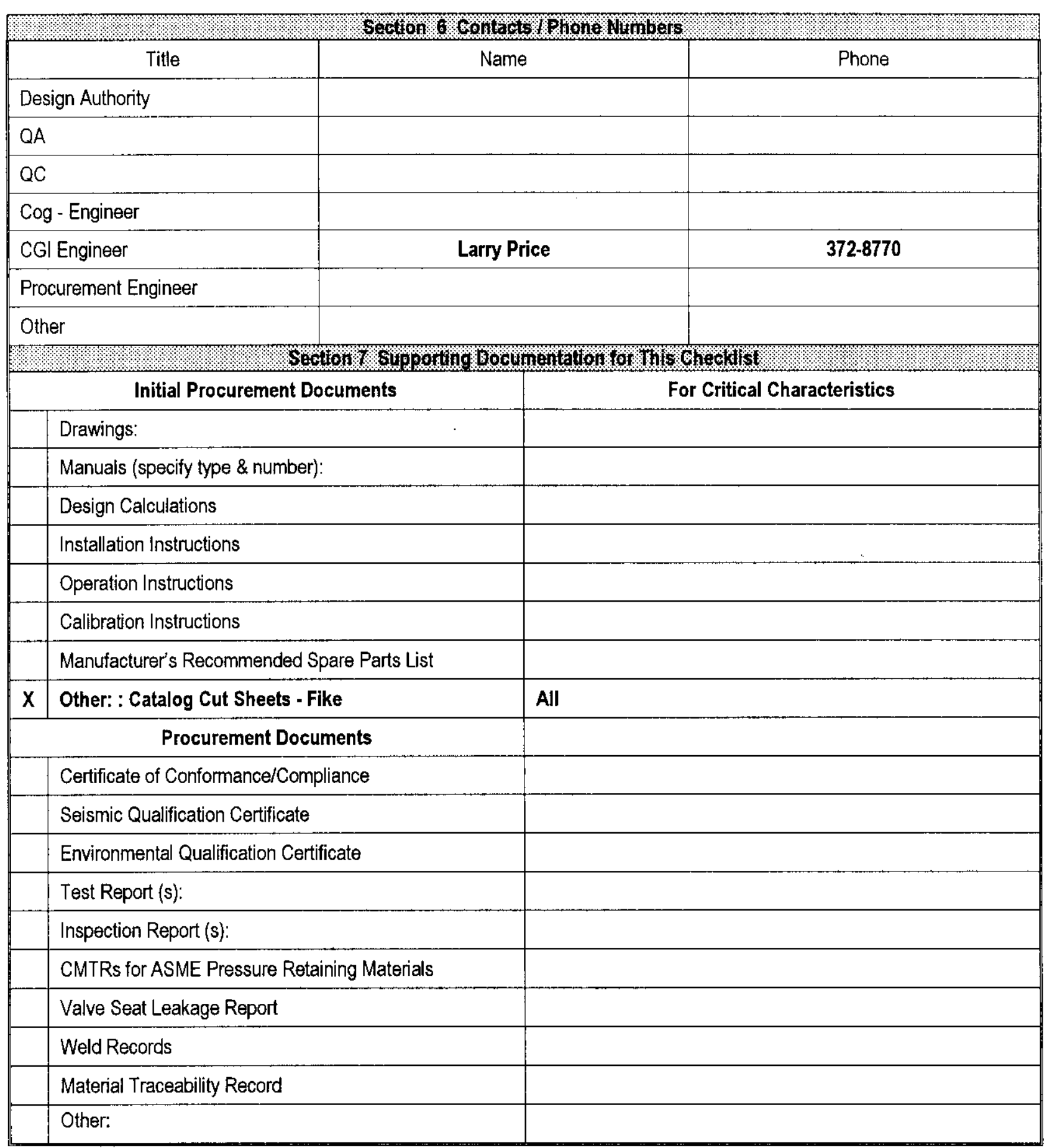




\section{DISTRIBUTION SHEET}

To

Distribution

Project Title/Work Order

W-441, SNF-3936, Rev. 2

Name

D. Whitehurst

G. Singh

A. Artzer (CVD Library)

R. Ramsgate

J. Brehm

P. Beaudet

P. Morrell (AVS)

M. Evarts (AI)

L. Price

SNE Startup

SNE Project Files

SNE Satelite Library

C. Van Katwijk

D. Whitworth

T. Nuxal1

C. Miska
From

T. Nuxal1, SNF-CVD
Page 1 of 1

Date $3 / 7 / 00$

EDT No. N/A

ECN No. 658711

Text With All Attach.

\begin{tabular}{l|l} 
MSIN & $\begin{array}{l}\text { With All } \\
\text { Attach. }\end{array}$
\end{tabular}

R3-86

R3-86

R3-86

R3-86

R3-26

S8-07

G1-50

$\mathrm{N} 1-29$

R3-26

B2 -64

R3-11

X3-25

R3-47

R3-11

R3-86

R3-86
Attach.I

Text Only Appendix

Only
EDT/ECN Only 